\title{
Intelligence of reconstructed biomolecular motor system
}

\author{
Daisuke Inoue \\ Faculty of Science, Hokkaido \\ Arif Md. Rashedul Kabir \\ Faculty of Science, Hokkaido \\ Akira Kakugo \\ University, Sapporo 060-0810, Japan \\ +81-11-706-3474 \\ University, Sapporo 060-0810, Japan \\ +81-11-706-3474 \\ Faculty of Science, Hokkaido \\ daisukeinoue@mail.sci.hokudai Kabir@mail.sci.hokudai.ac.jp \\ .ac.jp \\ University, Sapporo 060-0810, Japan \\ kakugo@sci.hokudai.ac.jp
}

\begin{abstract}
Collective motion is a fascinating example of coordinated behavior of self-propelled objects, which is often associated with the formation of large scale patterns. Nowadays, in vitro gliding assay is being considered a model system to experimentally investigate various aspects of group behavior and pattern formation by self-propelled objects. In this work, we have demonstrated the collective motion of kinesin driven microtubules by regulating mutual interaction among the gliding microtubules, by employing depletion force among them. Proper regulation of the mutual interaction among the gliding microtubules through employment of the depletion force was found to allow the exhibition of collective motion and stream pattern formation by microtubules. We also discuss how collectively moving microtubule on kinesin coated elastomer substrate response to external stimuli such as mechanical stresses. .
\end{abstract}

\section{Keywords}

Microtubule/Kinesin, Collective motion, stimuli-responsiveness

\section{INTRODUCTION}

Collective motion is a common display of coordinated behaviour which emerges from moving objects such as animal, birds, fishes, insects, bacteria, cells and self-propelled particles. One of the fundamental properties of collective motion is the evolution of fascinating large scale patterns, such as stream and vortices pattern. Recently, biomolecular motor systems such as Factin/myosin and microtubule/dynein have been used as model systems for experimentally demonstrating collective motion of self-propelled objects by employing them in the in vitro gliding assay where cytoskeletal filaments are driven by biomolecular motors immobilized on a surface in the presence of adenosine triphosphate (ATP) [1-6]. These experimental evidences have emphasized the importance of local interaction between gliding cytoskeletal filaments in the collective motion and pattern formation. Thus, the in vitro gliding assay offers a simple means to investigate experimentally roles of parameters that govern collective motion and pattern formation. However, the

investigation using biomolecular motor system was not always successful due to failure of biomolecular motor systems in exhibiting collective motion. To overcome this drawback, we for the first time demonstrate that regulation of local interaction among gliding microtubules allows them exhibit collective motion even on a kinesin coated surface. We have regulated the interaction of gliding microtubules by employing depletion force among them which is an attractive interaction known to work between colloidal particles or macromolecules suspended in polymer solution such as methylcellulose (MC) or polyethylene glycol (PEG). Depletion force mediated collective motion and subsequent pattern formation by microtubules on a kinesin coated substrate indicates that emergence of collective motion is independent of the type of biomolecular motor used in the in vitro gliding assay, if the interaction among the cytoskeletal filaments is properly regulated. This work offers a universal means for demonstrating the collective motion of biomolecular motor driven cytoskeletal protein filaments using the in vitro gliding assay, which in turn is expected to widen the applications of biomolecular motor systems and might foster the present understanding on coordinated behavior and pattern formation by self-propelled objects.

\section{Results and discussions [7]}

The effect of depletion force induced by MC on the assembly of microtubules was firstly investigated by monitoring the organization of microtubules suspended in MC solutions of different concentrations. Low concentration microtubules, prepared from $200 \mathrm{nM}$ tubulin, was suspended in 0.1 and $0.3 \mathrm{wt} \%$ MC (methylcellulose 4000, Junsei Chemical Co., Ltd, MW=140 $\mathrm{kDa}$ ) solutions and observed under fluorescence microscopy. At $0.1 \mathrm{wt} \%$ of MC solution, microtubules were found to form aster like aggregations. On further increasing the MC concentration to $0.3 \mathrm{wt} \%$, thick and long microtubule bundles were formed owing to increased attractive interaction among microtubules. In contrast, aggregation and bundle formation of microtubules were not observed in the absence of $\mathrm{MC}(0 \mathrm{wt} \%)$. This result indicates that depletion force induced by MC can effectively enhance the attractive interaction among microtubules and allow their assembly formation, which coincides well with the previous report [8].To investigate the effect of depletion force on the behaviour of gliding microtubules, we performed an in vitro gliding assay of microtubules in the presence of MC. GFP-tagged kinesin (recombinant conventional kinesin consisting of 560 amino acid of human kinesin-1) was immobilized on the glass substrate via anti-GFP antibody. Then microtubules were deposited at the kinesin coated glass surface. Motility of microtubules was initiated by adding ATP buffer at $25{ }^{\circ} \mathrm{C}$. Investigation on the behaviour of gliding microtubules filaments revealed that during movement, microtubules randomly approached each other and 
collided resulting in either snuggling or crossing over. During snuggling, gliding microtubules interacted showing a parallel or antiparallel alignment where the terms indicate that snuggling microtubules are aligned following same or opposite polarity, respectively. Snuggling is considered the most important behaviour of gliding microtubules for producing collective motion that often leads to formation of stream or vortex patterns of microtubules. Next, we investigated the behaviour of gliding microtubules in the presence of depletion force induced by MC. Here, we initiated the motility of microtubules by applying ATP buffer containing MC of two different concentrations ( 0.1 and 0.3 $\mathrm{wt} \%$ ). In the presence of $0.1 \mathrm{wt} \% \mathrm{MC}$, the probability of snuggling event was increased to $30 \%$ and increasing $\mathrm{MC}$ concentration further to $0.3 \mathrm{wt} \%$, the probability of snuggling also increased to $50 \%$. In these two cases, the probability of snuggling remained insensitive to the change in kinesin density on the substrate. To explore the condition for obtaining collective motion of microtubules at fixed kinesin and MC concentrations of 1000 $\mathrm{nM}$ and $0.3 \mathrm{wt} \%$, respectively, we investigated how the microtubule density affects their moving behaviour by varying the concentration of microtubules prepared by tubulin solution of 0.2 to $5.0 \mu \mathrm{M}$. Initially, just after addition of ATP (time set as $0 \mathrm{~min}$ ), the microtubules at any of the concentrations moved randomly without showing any specific directional preference. Over time ( 30 min), randomly moving microtubules showed collective motion which resulted in the formation of large streams. Collective motion of microtubules was observed above a certain concentration of microtubules, i.e. at a tubulin concentration of $2.0 \mu \mathrm{M}$. The size of streams was increased with increasing the concentration of microtubules. To evaluate emergence of collective motion, we quantified the orientation of microtubules by analysing fluorescence microscopy images of collectively moving microtubules at $30 \mathrm{~min}$. First, we obtained the distribution of orientation angles of gliding microtubules from which we calculated the nematic order parameter, $S$ which is the degree of orientation of microtubules. The $S=0$ and $\sim 1$ represent random and uniaxial orientation distribution of microtubules, respectively. There exists a critical density of microtubules, $\rho_{\mathrm{c}}$ at $\sim 28 \times 104$ mm-2 above which the $S$ remained constant and was close to 1 . This transition in $\mathrm{S}$ depending on microtubule concentration well represents a phase transition of liquid crystal from an isotropic to a nematic phase. To quantitatively characterise the change in orientation and density of microtubules as observed in this figure, we adopted two parameters: nematic order parameter, $S$ and density fluctuation, $D_{f}$. Here, $D_{f}$ is a parameter that can characterize the density fluctuation of microtubules in different gathering states. In the crowded state, microtubules locally form highly dense streams, whereas the scattered state is manifested by a reduction of numbers of microtubules in the streams over time. The $\mathrm{S}$ of microtubules initially increased with time but it decreased after $1 \mathrm{~h}$. This time-dependent change in the $\mathrm{S}$ reflects the change in orientation of microtubules with time and after $1 \mathrm{~h}$, alignment of microtubules started to be random. For the $\mathrm{D}_{\mathrm{f}}$, it increased due to the formation of local streams over time. We also investigated response of collectively moving microtubule network on kinesin coated elastomer substrate by employing uni-axial stretching stress. On application of uni-axial stretching stress, stream like ordered network are self-organized into various patterns such as perpendicular oriented and zigzag pattern depending on mode of stress. More detailed results will be reported in the presentation.

\section{Conclusion}

In conclusion, by employing a macromolecule (methylcellulose) induced depletion force, we demonstrated the first-ever collective motion and stream pattern formation by microtubules on a kinesin coated surface. This method offers a simple and universal technique to investigate the coordinated behaviour of selfpropelled objects using biomolecular motor systems. Consequently, this will be helpful in understanding not only the collective behaviour of self-propelled objects such as birds, animals or fishes, but also may provide new insight into emergent structures obtained through a non-equilibrium process. Recently microtubule/kinesin system has attracted attention in the field of molecular robotics as the smallest self-propelled objects. Molecular robots, relying on a large number of collectively moving self-propelled objects such as gliding microtubules, enables parallel processing in transporting a large number of small cargos and assembling building blocks into an ordered structure. Therefore, ideas obtained from the present study on collective motion of gliding microtubules are expected to expand the boundaries in the field of molecular robotics.

\section{ACKNOWLEDGMENTS}

This research was financially supported by a Grant-in-Aid forScientific Research on Innovative Areas "Molecular Robotics" (grant number 24104004) and Grant-in-Aid for JSPS Fellowsfrom the Japan Society for the Promotion of Science.

\section{REFERENCES}

[1] H. Hess, J. Clemmens, C. Brunner, R. Doot, S. Luna, K.-H. Ernst and V. Vogel, Nano Letters, 2005, 5, 629-633.

[2] R. Kawamura, A. Kakugo, K. Shikinaka, Y. Osada , J. P. Gong, Biomacromolecules, 2008, 9, 2277-2282.

[3] V. Schaller, C. Weber, C. Semmrich, E. Frey and A. R. Bausch, Nature 2010, 467, 73-77.

[4] V. Schaller, C. Weber, B. Hammerich, E. Frey and A. R. Bausch, Proc. Natl. Acad. Sci. USA, 2011, 108, 1918319188.

[5] V. Schaller, C. Weber, E. Frey and A. R. Bausch, Soft Matter, 2011, 7, 3213-3218.

[6] Y. Sumino, K. H. Nagai, Y. Shitaka, D. Tanaka, K. Yoshikawa, H. Chaté and K. Oiwa, Nature, 2011, 483, 448452.

[7] D. Inoue, B. Mahmot, Arif Md. Rashedul Kabir, T. Ishrat Farhana, K. Tokuraku, K. Sada, A. Konagaya, and A. Kakugo Nanoscale DOI: $10.1039 / \mathrm{c} 5 \mathrm{nr} 02213 \mathrm{~d}$

[8] S. Köhler, O. Lieleg and A. R. Bausch, PLoS ONE, 2008, 3, e2736. 